\title{
Report of a Type AB Thymoma with an Atypical Type A component
}

\author{
Anurita Kar ${ }^{1 *}$ and Archana Pandita ${ }^{2}$ \\ ${ }^{1}$ Anatomical Pathology Registrar, Waikato Hospital, New Zealand \\ ${ }^{2}$ Anatomical Pathology Consultant, Waikato Hospital, New Zealand
}

\section{Case Report}

Thymomas are rare tumours of the mediastinum, their cell of origin being thymic epithelial cells. They are defined as neoplasms of thymic epithelial cells with or without a background of non-neoplastic lymphocytes. Microscopic categorisation is broadly based on the presence or absence of lymphocytes followed by the cytologic attributes of the neoplastic epithelial cells [1].

With the variety of presentations and criteria for malignancy, it has always been challenging to classify them appropriately. The classification has evolved over the decades from Muller-HermelinkMarino classification scheme to the most current 2015 World Health Organisation (WHO) classification scheme [2].

In the current $\mathrm{WHO}$ classification, there are five main histological types of thymomas - A, AB, B1, B2, and B3. Atypical type A thymoma has been recently added to the WHO classification (in 2015) [1]. There are rare reports of atypical type $\mathrm{A}$ thymomas as well as $\mathrm{AB}$ thymomas with an atypical A component demonstrating cytologic atypia as well as foci of necrosis, increased mitoses et cetera. We report a case of type $\mathrm{AB}$ thymoma with an atypical A component that we came across in our institution recently (Figures 1-4).

An otherwise fit and healthy, 55 year old man had an incidental finding of a $13 \mathrm{~mm}$ mediastinal mass while having a CT scan for a skin lesion on his right shoulder. The CT scan was initially requested to ensure that there was no invasion of the skin lesion into the deeper structures. On questioning he only reported occasional cough with no history of haemoptysis or sputum production. There was no history of fever, night sweats or weight loss. Radiology showed a large cystic and calcified extrapulmonary intrathoracic mass compressing the right atrium. His CEA, HCG, AFP and LDH levels were reported as normal. A thymectomy was performed.

Macroscopically the specimen weighed $562 \mathrm{~g}$ and consisted of a firm mass with a soft, heterogenous, yellow-brown cut surface with cavitations. It measured $125 \mathrm{~mm}$ in maximum dimension. The mass appeared to be well encapsulated. Histology revealed a lobulated tumour with islands of spindle cell type $\mathrm{A}$ areas where there were minimal lymphocytes and areas rich in lymphocytes (type B). Perivascular spaces were not a feature. The areas of necrosis appeared infarct-like with foamy histiocytes and cholesterol clefts. Focally coagulative tumour necrosis and comedonecrosis was seen. Type A areas also showed increased mitoses (up to 6 mitoses per $2 \mathrm{~mm}^{2}$ )

On Immunohistochemistry, the tumour cells were positive for Pan-CK, BCL-2, CK 5/6, CK14, p63 with a patchy CK7 positivity. The tumour cells were negative for CD5 and CD117. The overall features

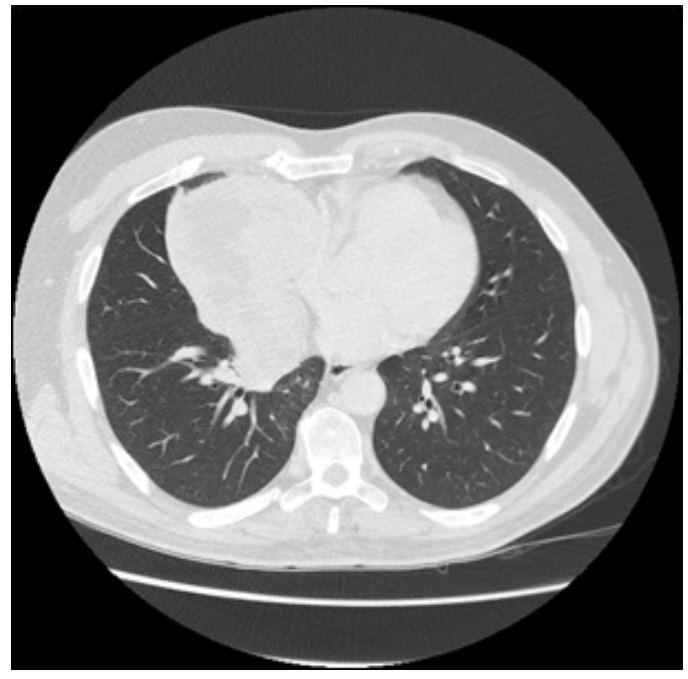

Figure 1. CT image of Thymoma.

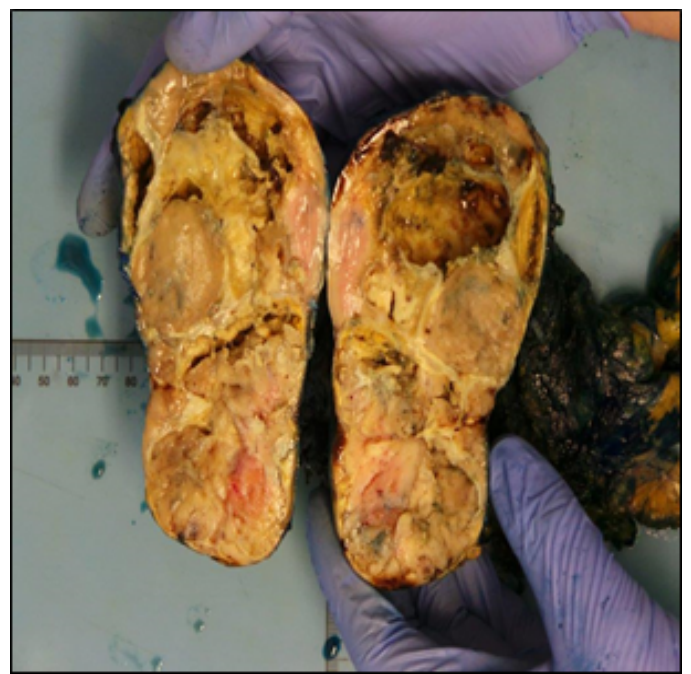

Figure 2. Macroscopic image of Thymoma.

${ }^{\star}$ Correspondence to: Anurita Kar, Anatomical Pathology Registrar, Waikato Hospital, New Zealand, E-mail: anurita.kar@waikatodhb.health.nz

Key words: thymoma, mediastinal mass, Atypical Type A component

Received: June 10, 2020; Accepted: June 18, 2020; Published: June 25, 2020 


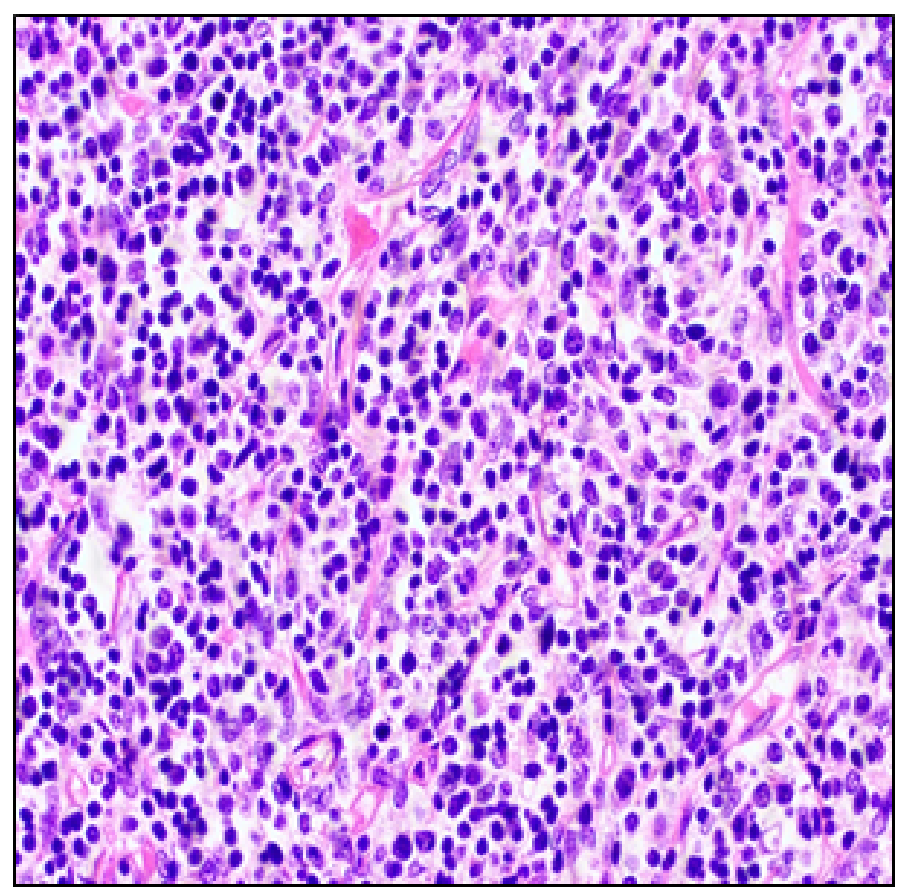

Figure 3. Histology of Thymoma - Type AB component.

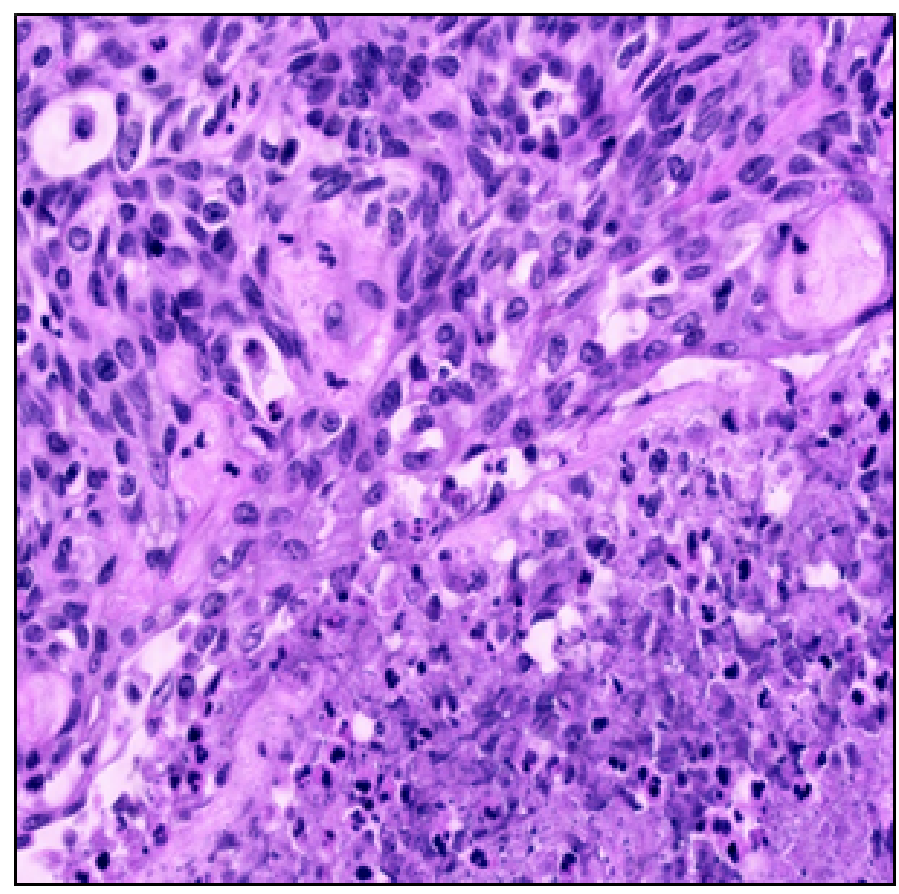

Figure 4. Histology of Thymoma - Atypical Type A Component.

suggested a type $\mathrm{AB}$ thymoma with an atypical type $\mathrm{A}$ component. This was also reviewed and confirmed by Dr N Kingston, Auckland Hospital.

All thymomas are potentially malignant however in the lower grade tumours local recurrence or extension into adjacent structures is more common as compared to the high-grade tumours where distant metastases have been reported [1,3].

Type $\mathrm{AB}$ thymomas are denoted as mixed thymomas containing both type $A$ and type $B$ areas. Both type $A B$ and type $A$ thymomas are generally lower grade with risk of local recurrence. The new thymoma variant, atypical type A thymoma is similar in appearance to type A thymomas in that it has spindled to oval shaped cells with few/ sparse lymphocytes. However, in contrast to type A thymomas they demonstrate cytologic atypia. The WHO classification of 2015 defines it as featuring spindle to oval shaped cells with mild to moderate nuclear atypia, hypercellularity, increased pleomorphism and nuclear hyperchromasia as well as foci of necrosis or comedonecrosis. It also has an increased mitotic count of $>4$ per $2 \mathrm{~mm}^{2}$. All of these features are traditional features of malignancy or likely to denote a more aggressive course [1].

The usual prognostic markers for distant spread are higher stage and positive surgical margins. There have been few reports of metastatic carcinoma to lung and brain where the tumour demonstrates atypical features to a variable degree. The metastases have been reported even up to 15 years from the time of diagnosis [3]. However, on extensive literature search there was a paucity of articles on atypical A Thymoma, their overall clinical course and prognosis.

The case reports reported in the literature show a more aggressive course of atypical type A thymoma in comparison to type A or type AB. Grajkowska et al. reported a similar case of type $A B$ thymoma with an atypical A component showing distant metastasis to the lung and brain, 10 and 15 years respectively after the original surgery. Microscopically the metastases resembled the atypical A component of the tumour [3].

Vladislav et al. in their case report series described the presence of tumour necrosis as being an indicator of future relapse including distant metastases in spite of a successful initial surgery with negative surgical margin [4]. Currently there is no standard therapeutic method of approach for patients with metastatic thymoma and it is unclear whether neoadjuvant chemotherapy or radiotherapy would confer a survival advantage or prevention of future recurrence. Given the reports of clinically aggressive behaviour in a few cases with atypical type A thymoma (especially those in which necrosis is seen) careful and extended postoperative follow up is required examining both the intrathoracic and extrathoracic cavities [5].

In the case of our patient, he is currently well and under careful post-operative follow up. We conclude that more research and study is required to further define the clinical course and overall prognosis of cases of thymomas with an atypical component.

\section{References}

1. Marx A, Chan JKC, Coindre JM, Detterbeck F, Girard N, et al. (2015) The 2015 World Health Organization Classification of Tumors of the Thymus: Continuity and Changes. J Thorac Oncol 10: 1383-1395. [Crossref]

2. Marino M, Roden AC (2018) The evolution of the histopathologic classification of thymic epithelial tumors. Mediastinum 2: 9

3. Grajkowska W, Matyja E, Kunicki J, Szymanska S, Marx A, et al. (2017) AB thymoma with atypical type A component with delayed multiple lung and brain metastases. $J$ Thorac Dis 9: E808-E814. [Crossref]

4. Vladislav IT, Gökmen-Polar Y, Kesler KA, Loehrer PJ, Badve S (2013) The role of histology in predicting recurrence of type A thymomas: a clinicopathologic correlation of 23 cases. Mod Pathol 26: 1059-1064.

5. Hashimoto M, Shimizu S, Takuwa T, Tsukamoto Y, Tsujimura T, et al. (2016) A case of atypical type A thymoma variant. Surg Case Rep 2: 116. [Crossref]

Copyright: (C2020 Kar A. This is an open-access article distributed under the terms of the Creative Commons Attribution License, which permits unrestricted use, distribution, and reproduction in any medium, provided the original author and source are credited. 